\title{
Hawking radiation is corpuscular
}

\author{
Wolfgang Mück ${ }^{1,2, a}$ \\ ${ }^{1}$ Dipartimento di Fisica “Ettore Pancini”, Università degli Studi di Napoli "Federico II", Via Cintia, 80126 Naples, Italy \\ ${ }^{2}$ Istituto Nazionale di Fisica Nucleare, Sezione di Napoli, Via Cintia, 80126 Naples, Italy
}

Received: 13 June 2016 / Accepted: 24 June 2016 / Published online: 5 July 2016

(c) The Author(s) 2016. This article is published with open access at Springerlink.com

\begin{abstract}
The total number of Hawking quanta emitted during the evaporation of a Schwarzschild black hole is proportional to the square of the initial mass or, equivalently, to the Bekenstein entropy. This simple, but little appreciated, fact is interpreted in terms of the recent discovery of black hole soft hair.
\end{abstract}

\section{Estimate of the number of Hawking particles}

In 1974, Hawking discovered that black holes emit radiation with a thermal power spectrum [1,2]. Soon after, Page gave an estimate of the life-time of a black hole from the knowledge of the emitted power [3]. In a simplified version of this calculation, one assumes that the black hole, at any value of the temperature, can be considered as an ideal black body emitting radiation in the form of massless bosons. As the black hole evaporates, its temperature is assumed to increase adiabatically as a function of the remaining mass. This simplification omits the gray-body factors [4], superradiance, and much of the backreaction of the radiation on the geometry.

The purpose of this short note is to provide an estimate of the total number of quanta emitted by the black hole. That number turns out to be proportional to the square of the black hole's initial mass in Planck units, which is reminiscent of the proposed graviton number in the black hole quantum $N$ portrait $[5,6]$. The simplicity of the calculation suggests that the result may be known, but I am not aware of it in the literature. ${ }^{1}$

\footnotetext{
a e-mail: wolfgang.mueck@na.infn.it
}

The spectral luminosity density of an ideal black body is derived from Planck's formula [8] and reads ${ }^{2}$

$\frac{\mathrm{d} L}{\mathrm{~d} \omega}=\frac{g A}{8 \pi^{2}} \frac{\omega^{3}}{\mathrm{e}^{\omega / T}-1}$,

where $A$ is the surface area, $T$ the temperature and $g$ the number of radiating degrees of freedom. ${ }^{3}$ The luminosity is obtained integrating (1) over the energy $\omega,{ }^{4}$

$L=\frac{g \pi^{2}}{120} A T^{4}$

Instead of the luminosity, one may also consider the emission rate (number flux) of the emitted quanta. The number flux density is related to the luminosity density by

$\frac{\mathrm{d} \Gamma}{\mathrm{d} \omega}=\frac{1}{\omega} \frac{\mathrm{d} L}{\mathrm{~d} \omega}=\frac{g A}{8 \pi^{2}} \frac{\omega^{2}}{\mathrm{e}^{\omega / T}-1}$.

Integrating (3) over the energy, one finds the emission rate

$\Gamma=\frac{g \zeta(3)}{4 \pi^{2}} A T^{3}$

where $\zeta(x)$ denotes the Riemann zeta function.

Now consider a Schwarzschild black hole of mass $M$, with Hawking temperature and horizon area given by

$T=\frac{\kappa}{2 \pi}=\frac{1}{8 \pi M}, \quad A=4 \pi r_{s}^{2}=16 \pi M^{2}$,

\footnotetext{
${ }^{1}$ While the manuscript was under revision, I learned from AlonsoSerrano that the calculation was performed recently [7].

2 Units are such that $\hbar=G=k_{B}=c=1$.

${ }^{3}$ E.g., $g=1$ for a spinless boson, while $g=2$ for photons and gravitons.

${ }^{4}$ For photons, this is the Stefan-Boltzmann law.
} 
respectively. Because the emitted power corresponds to the mass loss rate, (2) and (5) can be combined into

$\frac{\mathrm{d} M}{\mathrm{~d} t}=-L=-\frac{g}{15 \cdot 2^{11} \pi M^{2}}$

and one easily obtains the estimate for the evaporation time of the black hole,

$t_{\mathrm{ev}}=\frac{5 \cdot 2^{11} \pi}{g} M_{0}^{3}$.

where $M_{0}$ is the initial mass.

The emission rate of Hawking quanta is found by substituting (5) into (4),

$\Gamma=\frac{g \zeta(3)}{128 \pi^{4}} \frac{1}{M}$

Since Page's paper, the emission rate has appeared repeatedly in the literature; see, e.g., the recent references $[9,10]$ and references therein. It is very surprising that the total number of Hawking quanta emitted during the evaporation appears to have never been considered. Combining (8) with (6), one finds

$\frac{\mathrm{d} N}{\mathrm{~d} M}=\frac{\Gamma}{\frac{\mathrm{d} M}{\mathrm{~d} t}}=-\frac{240 \zeta(3)}{\pi^{3}} M$.

A simple integration yields the total number of Hawking quanta

$N=\frac{120 \zeta(3)}{\pi^{3}} M_{0}^{2}$

which is equal, up to a numerical factor, to the initial Bekenstein entropy

$N=\frac{30 \zeta(3)}{\pi^{4}} S$

independently of the number of radiating degrees of freedom.

The semi-classical analysis remains valid as long as $M \gg$ 1. The quantum regime may be modeled by an ultraviolet cutoff on the energy and mass integrals and gives rise to $1 / \mathrm{N}$ corrections. Hence, for a macroscopic black hole, the final stage of the evaporation, which happens in a quantum gravity regime, appears to be irrelevant in terms of the emitted Hawking quanta and of the information they carry. The inclusion of gray-body factors will likely affect the numerical prefactor in (10) and (11).

\section{Conclusions}

The above results appear to be relevant for recent developments on the physics of black holes and, in particular, for the information paradox. First, (10) is recognized as the proposed number of gravitons in the black hole quantum $N$-portrait $[5,6]$. In this proposal, a black hole is pictured as a BoseEinstein condensate of a very large number, $N \sim M^{2}$, of gravitons at the verge of a quantum phase transition. Hawking radiation is the result of the depletion of the condensate caused by 2-body interactions and contains non-thermal features of order $1 / N$, which resolve the information paradox $[11,12]$. The results (10) and (11) provide direct evidence in support of the quantum $N$-portrait, in the sense that a semiclassical black hole may be considered as a bound state of the $N$ Hawking particles it dissolves into. Interpreting each emitted Hawking particle as an information-carrying unit, (11) is indeed the expected relation between the entropy and the particle number. This indicates that it is more appropriate to give Hawking radiation, which is sparse in the semiclassical regime [10], a corpuscular interpretation instead of an undulatory one.

Second, the results should be interpreted in the light of recent developments on the infrared structure of quantum gravity, which revived results of Bondi, van der Burg, Metzner and Sachs (BMS) [13,14] from the early 1960s. Soft symmetries, or better, the supertranslations of the extended BMS symmetry group $[15,16]$, give rise to conserved charges [17-21] of the gravitational $S$-matrix. These soft supertranslation charges constitute what has been known as gravitational memory. Extending these results to the asymptotic symmetries of the black hole horizon [22-24], it has been discovered that black holes carry 'soft hair' [25], which retains the information as regards the state before the black hole formation and imprints that information, as the black hole evaporates, on the outgoing Hawking radiation. Classically, the information storage capacity of the horizon is infinite, but it is physically impossible to excite soft quanta that are smaller than the Planck area on the horizon, giving rise to an effective pixelization in agreement with the Bekenstein entropy-area law. Equations (10) and (11) provide evidence for this in the evaporation process. The information as regards the final state is measurable by the gravitational memory effect, i.e., the soft supertranslation charges at future null infinity that are carried by the outgoing Hawking quanta. This points again at the importance of the corpuscular point of view of Hawking radiation. The effective, semi-classical, gravitational memory capacity used by the Hawking quanta is of order $N \sim M_{0}^{2}$. Hence, it appears that unitarity is preserved, after all, when a gravitational system of total mass $M_{0}$ collapses into a black hole and evaporates subsequently. 
Acknowledgments I would like to thank R. Casadio for helpful discussions and his encouragement to publish this trivial calculation. Moreover, I am grateful to A. Alonso-Serrano for advising me of Ref. [7]. This research is supported in part by the INFN, research initiative STEFI.

Open Access This article is distributed under the terms of the Creative Commons Attribution 4.0 International License (http://creativecomm ons.org/licenses/by/4.0/), which permits unrestricted use, distribution, and reproduction in any medium, provided you give appropriate credit to the original author(s) and the source, provide a link to the Creative Commons license, and indicate if changes were made.

Funded by SCOAP ${ }^{3}$.

\section{References}

1. S.W. Hawking, Nature 248, 30 (1974). doi:10.1038/248030a0

2. S.W. Hawking, Commun. Math. Phys. 43, 199(1975). doi:10.1007/ BF02345020. [167(1975)]

3. D.N. Page, Phys. Rev. D 13, 198 (1976). doi:10.1103/PhysRevD. 13.198

4. J.B. Bekenstein, A. Meisels, Phys. Rev. D 15, 2775 (1977)

5. G. Dvali, C. Gomez, Fortsch. Phys. 61, 742 (2013). doi:10.1002/ prop. 201300001

6. G. Dvali, C. Gomez, Eur. Phys. J. C 74, 2752 (2014). doi:10.1140/ epjc/s10052-014-2752-3

7. A. Alonso-Serrano, M. Visser (2015). arXiv:1512.01890 [gr-qc]

8. M. Planck, Annalen der Physik 4, 553 (1901)

9. M. Visser, JHEP 07, 009 (2015). doi:10.1007/JHEP07(2015)009

10. F. Gray, S. Schuster, A. Van-Brunt, M. Visser, Class. Quant. Grav. 33(11), 115003 (2016). doi:10.1088/0264-9381/33/11/115003
11. G. Dvali, C. Gomez, Phys. Lett. B 719, 419 (2013). doi:10.1016/j. physletb.2013.01.020

12. G. Dvali, Fortsch. Phys. 64, 106 (2016). doi:10.1002/prop. 201500096

13. H. Bondi, M.G.J. van der Burg, A.W.K. Metzner, Proc. R. Soc. Lond. A 269, 21 (1962). doi:10.1098/rspa.1962.0161

14. R.K. Sachs, Proc. R. Soc. Lond. A 270, 103 (1962). doi:10.1098/ rspa.1962.0206

15. G. Barnich, C. Troessaert, Phys. Rev. Lett. 105, 111103 (2010). doi:10.1103/PhysRevLett.105.111103

16. G. Barnich, C. Troessaert, JHEP 12, 105 (2011). doi:10.1007/ JHEP12(2011)105

17. A. Strominger, JHEP 07, 152 (2014). doi:10.1007/ JHEP07(2014)152

18. T. He, V. Lysov, P. Mitra, A. Strominger, JHEP 05, 151 (2015). doi:10.1007/JHEP05(2015)151

19. A. Strominger, A. Zhiboedov, JHEP 01, 086 (2016). doi:10.1007/ JHEP01(2016)086

20. D. Kapec, V. Lysov, S. Pasterski, A. Strominger, JHEP 08, 058 (2014). doi:10.1007/JHEP08(2014)058

21. E.E. Flanagan, D.A. Nichols (2015). arXiv:1510.03386 [hep-th]

22. L. Donnay, G. Giribet, H.A. Gonzalez, M. Pino, Phys. Rev. Lett. 116(9), 091101 (2016). doi:10.1103/PhysRevLett.116.091101

23. G. Dvali, C. Gomez, D. Lüst, Phys. Lett. B 753, 173 (2016). doi:10. 1016/j.physletb.2015.11.073

24. M. Hotta, J. Trevison, K. Yamaguchi (2016). arXiv:1606.02443 [gr-qc]

25. S.W. Hawking, M.J. Perry, A. Strominger, Phys. Rev. Lett. 116(23), 231301 (2016). doi:10.1103/PhysRevLett.116.231301 